\title{
Sleep problems in university students - an intervention
}

\author{
Angelika Anita Schlarb* \\ Anja Friedrich* \\ Merle Claßen \\ Faculty of Psychology and Sports, \\ Bielefeld University, Bielefeld, \\ Germany \\ *These authors contributed equally \\ to this work
}

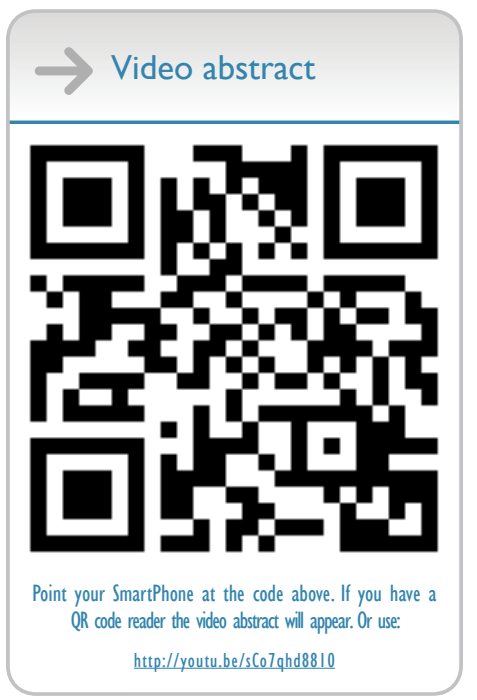

Correspondence: Angelika Anita Schlarb Faculty of Psychology and Sports, Bielefeld University, Universitätsstraße 25, 33615 Bielefeld, Germany Tel +4952I 1066962

Email angelika.schlarb@uni-bielefeld.de

\author{
This article was published in the following Dove Press journal: \\ Neuropsychiatric Disease and Treatment \\ 26 July 2017 \\ Number of times this article has been viewed
}

Introduction: Up to $60 \%$ of all college students suffer from a poor sleep quality, and $7.7 \%$ meet all criteria of an insomnia disorder. Sleep problems have a great impact on the students' daily life, for example, the grade point average. Due to irregular daytime routines, chronotype changes, side jobs and exam periods, they need specialized treatments for improving sleep. "Studieren wie im Schlaf" (SWIS; (studying in your sleep)) is a multicomponent sleep training that combines Cognitive Behavioral Therapy for Insomnia and Hypnotherapy for Insomnia to improve students' sleep, insomnia symptoms and nightmares. The aim of the present study is to evaluate the acceptance, feasibility and the first effects of SWIS.

Methods: Twenty-seven students (mean $=24.24$, standard deviation $=3.57$ ) participated in a study of pre-post design. The acceptance and feasibility were measured with questionnaires. In addition, the Pittsburgh Sleep Quality Index (PSQI), sleep logs and actigraphy were implemented. Further variables encompassed daytime sleepiness, sleep-related personality traits and cognitions about sleep.

Results: Seventy-four percent of the participants reported symptoms of an insomnia disorder, and $51.9 \%$ fulfilled all criteria of an insomnia disorder according to the Diagnostic and Statistical Manual of Mental Disorders (fifth edition). Correspondingly, the students suffered from clinically relevant sleep problems according to the PSQI. The SWIS sleep training is a well-accepted and feasible program. Significant improvements were observed in the subjective sleep quality and sleep-related personality traits, as well as clinical improvements in objective sleep measures.

Discussion: Findings showed that SWIS is a feasible program for the treatment of sleep problems in college and university students due to its various effects on sleep and cognitive outcomes. Further evaluation of follow-up measurements and additional variables, that is, cognitive performance and mental health, is needed.

Keywords: sleep quality, insomnia, nightmares, college students, CBT-I, HT-I

\section{Introduction}

Students experience several important developments when starting at university. They have to cope with "leaving home, increased independence, changes in peer groups, new social situations, maintenance of academic responsibilities and increased access to alcohol or drugs". ${ }^{1}$ About $90 \%$ of university students have roommates, and among them, $41 \%$ wake up at night due to the noise of others. Bed- and risetimes on weekdays and weekends often differ in the range of more than 1 to 2 hours. These challenges and special circumstances faced by university students are associated with sleep disturbances. ${ }^{2}$ About $60 \%$ suffer from a poor sleep quality according to the PSQI. ${ }^{3}$ Gaultney revealed that $27 \%$ of all university students are at a risk of at least one sleep disorder. ${ }^{4}$ Furthermore, previous findings reported that a minimum $7.7 \%$ of students suffer from insomnia and $24.3 \%$ from nightmares. ${ }^{5,6}$ 
Sleep problems and sleep disorders severely impair university students' academic success. In a study conducted by Buboltz et al, $31 \%$ of all students suffered from morning tiredness. ${ }^{2}$ In another study, poor sleepers reported reduced daytime functioning. ${ }^{7}$ Shorter sleep duration and an irregular sleep-wake schedule significantly correlated with a lower GPA. ${ }^{4}$ Regarding sleep habits, the wake-up times explained significant amounts of GPA variance. ${ }^{8}$ A clinical review provided evidence that sleep problems correlated with impeded learning, especially poorer declarative and procedural learning, neurocognitive performance and academic success. ${ }^{9}$

Beyond academic considerations, sleep problems in university students are often connected with mental health issues. It is common for students with insomnia to suffer from mental health problems such as chronic fatigue, depression, stress, lower optimism, anxiety and a lower quality of life. ${ }^{1,10,11}$ Taylor et al controlled the correlation between insomnia and mental health problems for medical problems (ie, migraines, gastrointestinal diseases, sexually transmitted diseases). Even then, significant correlations were found for obsessive-compulsive symptoms, somatization, depression, anxiety and overall symptoms. ${ }^{12}$ In addition, students suffering from insomnia often thought about their sleep problems or ruminated about daily events. ${ }^{13}$ Furthermore, students with worse sleep reported more rumination about a stressful life event (ie, exam).${ }^{14}$ Correspondingly, other studies showed that rumination predicted longer sleep-onset latency and was associated with lower sleep efficiency, wakefulness after sleep onset and reduced sleep quality. ${ }^{15,16}$ These associations also existed after controlling for general cognitive arousal, depression and anxiety. ${ }^{17,18}$ Vail-Smith et al discovered that students who reported physical aggression, suicide ideation and substance abuse had a significantly worse sleep quality than those who did not suffer from one of the aforementioned factors. ${ }^{19}$ This relationship between sleep problems and suicidality persisted when taking aggressive behavior and depression into account. ${ }^{20}$ Correspondingly, nightmares were significantly related to suicidal ideation even after controlling for depression, anxiety and posttraumatic stress disorder. ${ }^{21}$ Students who suffered from nightmares often reported more insomnia symptoms and lower self-efficacy. ${ }^{6}$

A poor sleep quality is often associated with stimulant use in university students. ${ }^{22}$ Correspondingly, sleep medication is the second most common substance group consumed by German university students. ${ }^{23}$

Finally, one-quarter of all university students are evening chronotypes. ${ }^{24}$ Eveningness is often associated with a poor sleep quality, ${ }^{25}$ lower self-control, more procrastination, ${ }^{26}$ more stress sensitivity ${ }^{27-29}$ and lower sleep efficiency. ${ }^{24}$ All in all, various sleep-related parameters and disorders affect university students' life.

Despite the high prevalence rates and the severe consequences, only a few studies examined the treatment of sleep disorders in university students. A recent systematic review found that there is a lack of specialized treatment of insomnia for college students. ${ }^{30}$ Cognitive-behavioral therapy was the most effective approach to improve sleep in university students with relaxation techniques, mindfulness and hypnotherapy additionally benefiting mental health outcomes. ${ }^{30}$ However, most of the 27 included studies were conducted on healthy students who did not suffer from sleep problems, different outcomes were investigated, objective sleep was ignored and only a handful of studies provided follow-up data. ${ }^{30}$ Not a single study examined other sleep disorders besides insomnia or assessed the sleep regularity and chronotype of the students.

\section{SWIS - a sleep training for university students}

"Studieren wie im Schlaf" (SWIS; studying in your sleep) - a sleep training for university students with sleep problems was derived from the structure of sleep treatment programs for different age groups, for example, Mini-KiSS ${ }^{31}$ for infants under the age of five, $\mathrm{KiSS}^{32}$ for children aged five to ten and $\mathrm{JuSt}^{33}$ for adolescents. With a maximum of six sessions, all these programs were relatively short and were based on a combination of CBT-I and HT-I. Correspondingly, the six SWIS sessions each contained CBT-I and HT-I elements. As a multicomponent intervention, SWIS focused on the challenges of university students' life by addressing special circumstances and environmental factors as well as biological factors. These included irregular sleep-wake cycles due to variability in their daily routine, side jobs, chronotype changes as well as learning and exam periods.

In this pilot study, we first examined the acceptance of participants and trainers. Secondly, we assessed whether the students and trainers rated SWIS as a feasible program. Thirdly, we investigated if the SWIS treatment improved various sleep variables including, a) symptoms of sleep disorders, b) sleep quality, c) daytime sleepiness, d) rumination and focusing, as well as e) dysfunctional cognitions about sleep. While the first two hypotheses aimed to evaluate the participants' and trainers' acceptance, the other hypothesis tested first hints toward effectiveness, thus incorporating both aspects of feasibility. 


\section{Methods}

\section{Design}

The quasi-experimental study investigated the acceptance and feasibility of SWIS. Four treatment groups with five to seven participants completed the training. All participants suffered from symptoms of sleep disorders, such as insomnia disorder, nightmares or irregular sleep-wake type, according to DSM-5. ${ }^{34}$ Two psychologists executed the training sessions during the afternoon or early evening. Every training session was supervised for manual adherence and treatment fidelity.

Sleep disorders were diagnosed according to the classification criteria of the DSM-5. ${ }^{34}$ In addition, participants were classified as "poor sleepers" by the PSQI if they scored above a cut-off of 5 , or as severely impaired sleepers if they scored above $10.35,36$

The pretest was taken 2 weeks before the treatment, and the post-measurement 2 weeks after the training. All participants provided written informed consent prior to diagnosis and treatment. The intervention involved six sessions. During pre- and post-measurement, participants filled out sleep logs for 14 days and wore actigraphs for 8 days to assess subjective and objective sleep quality. Furthermore, participants completed various questionnaires. The study was approved by the ethics committee of Bielefeld University.

\section{Inclusion criteria}

Based on the inclusion criteria, participants who fulfilled various criteria for an insomnia disorder or an irregular sleepwake type according to DSM-5 classification, ${ }^{34}$ as well as participants who reported nightmares (Table 1) were included in this study. Sleep was assessed with the self-developed SPQ. In addition, participants were categorized as "poor sleepers" by the PSQI. ${ }^{35}$

\section{Sleep Problems Questionnaire}

Participants received a 12-item SPQ (Table 2) based on the DSM-5 classification for an insomnia disorder and an

Table I Diagnostic criteria and SPQ

\begin{tabular}{ll}
\hline & Items \\
\hline Difficulties initiating sleep & SPQ I \\
Difficulties maintaining sleep & SPQ 2 \\
Early awakening & SPQ 3 \\
Subjective impairment & SPQ 4 \\
Insomnia & SPQ I/SPQ 2/SPQ 3+ SPQ 4 + \\
& SPQ 5 + SPQ 6 \\
Irregular sleep-wake type & SPQ 7/SPQ 9 + SPQ 4 + SPQ 5 + SPQ 6 \\
Nightmare & Nightmares at least three times per week \\
\hline Note: Slashes indicate additional choices (item I and/or item 2), whereas plus signs \\
indicate mandatory items (item I and item 2). \\
Abbreviation: SPQ, Sleep Problems Questionnaire.
\end{tabular}

Table 2 Sample and sleep disturbances during baseline

Pre-measurement

\begin{tabular}{ll}
\hline Sample characteristics & \\
N & 27 \\
Age, M (SD) (years) & $24.24(3.57)$ \\
Age range (years) & $20-35$ \\
Women (\%) & $21(77.8)$ \\
Men (\%) & $6(22.2)$ \\
Sleep disturbances (\%) & \\
Difficulties initiating sleep & 59.3 \\
Difficulties maintaining sleep & 59.3 \\
Early awakening & 51.9 \\
Subjective impairment & 66.7 \\
Insomnia disorder & 51.9 \\
Nightmares & 18.5 \\
Irregular sleep-wake type & 11.1
\end{tabular}

Abbreviations: $M$, mean; SD, standard deviation.

irregular sleep-wake type.$^{34}$ Due to the fact that the inclusion criteria questionnaire was developed specifically for this study, no psychometric properties were examined. Three items pertained to the diagnosis of insomnia disorder (ie, "I suffer because I have difficulties initiating sleep", items 1-3), two items supported an irregular sleep-wake-type diagnosis (ie, "I sleep at least three times in 24 hours", items 7,9 ) and the other six items determined sleep problem frequency (items 5, 6), impairment (item 8) and exclusion criteria ("My sleep problems are not mainly caused by bad sleep hygiene", items 10-12). The exclusion criteria were formulated in a double-negative form. A dichotomous answer mode (yes/no) was employed.

\section{Diagnostic criteria}

Insomnia was diagnosed if participants had trouble initiating sleep, woke up during the night, or had difficulties falling asleep after waking up at night. In addition, they had to report daytime impairments. Insomnia with sleep-onset latency was diagnosed if students fulfilled all criteria of an insomnia disorder and reported a sleep latency greater than 30 minutes. The irregular sleep-wake type included participants with fragmented sleep (at least three sleep periods in 24 hours) or other self-reported irregular sleep. To receive a diagnosis, the sleep problems had to cause significant distress, occur at least three times per week and persist for longer than 1 month. Nightmares were indicated if participants reported nightmares at least three times per week according to DSM-5 criteria (Table 1).

\section{Sample}

Figure 1 provides an overview of the participant flow during the study. Forty applications were screened, and 27 participants 


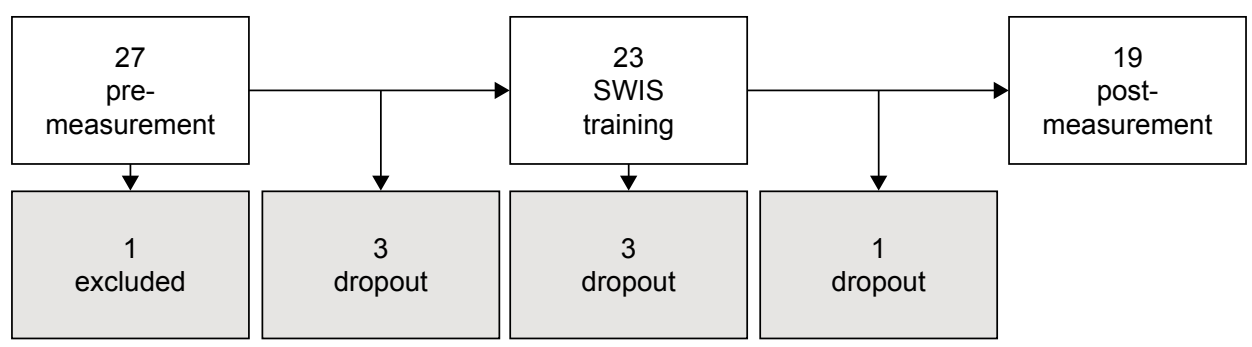

Figure I SWIS flowchart.

Abbreviation: SWIS, Studieren wie im Schlaf (studying in your sleep).

were invited to take part in the study. Due to exclusion (one participant) and dropouts before (three participants) and during the training (three participants) as well as dropouts after the training (one participant), 19 participants remained for the post-measurement.

The sample characteristics for the different measurement times are displayed in Table 3. The mean age was $\mathrm{M}=24.24$ $(\mathrm{SD}=3.57)$ years during pre-measurement. There were more women (77.8\%; men $22.2 \%)$ participants. One participant had to be excluded due to a low PSQI score (PSQI =4). The remaining 26 participants (96.29\%) scored above the PSQI cut-off defining a poor sleep quality (PSQI sum score $>5$ ).

Approximately two-thirds of the sample reported difficulties initiating sleep and maintaining sleep and subjective impairment because of the sleep problems. Of all the participants, $51.9 \%$ reported early awakening. While more than half of the students fulfilled all criteria for an insomnia disorder, nightmares and irregular sleep-wake types were less common.

Table 3 SAQ scales and items

\begin{tabular}{|c|c|c|}
\hline Scale & No & Items \\
\hline \multirow[t]{3}{*}{ Content } & I & The topic of today's session appealed to me \\
\hline & 4 & I received answers to my questions \\
\hline & 13 & The new information was helpful \\
\hline \multirow[t]{4}{*}{ Didactics } & 2 & $\begin{array}{l}\text { The theoretical and practical knowledge } \\
\text { was conveyed coherently }\end{array}$ \\
\hline & 3 & $\begin{array}{l}\text { My individual situation was } \\
\text { considered adequately }\end{array}$ \\
\hline & 6 & Enough opportunities for practice were provided \\
\hline & 9 & $\begin{array}{l}\text { Enough opportunities for group discussion } \\
\text { were provided }\end{array}$ \\
\hline \multirow[t]{3}{*}{ Transfer } & 5 & I gained new ideas for everyday life \\
\hline & 7 & $\begin{array}{l}\text { I received assistance for implementation } \\
\text { into everyday life }\end{array}$ \\
\hline & 10 & I will implement the strategies of today's session \\
\hline Hypnotherapy & 14 & The trance session was helpful \\
\hline \multirow[t]{2}{*}{ Trainer aptitude } & 11 & The trainer appeared to be competent \\
\hline & 12 & I felt understood by the trainer \\
\hline Group dynamics & 8 & I felt comfortable in the group \\
\hline
\end{tabular}

Abbreviation: SAQ, Student Acceptance Questionnaire.

\section{Instruments}

\section{Acceptance}

To evaluate the acceptance of the training, two questionnaires were implemented: one for the students (SAQ) and the other for the trainers (TAQ). They were based on similar questionnaires in a previous sleep study. ${ }^{33}$ Students and trainers anonymously completed the questionnaires after each session. Answering modes comprised a five-point Likert scale ranging from "applies to me completely" (1) to "does not apply to me at all" (5). Lower scores indicated a higher satisfaction with the sleep training.

\section{Student Acceptance Questionnaire}

The SAQ consisted of 14 items (Table 4). Four scales measured the participants' acceptance of each training session's content, didactics, transfer into everyday life and trainer aptitude. Two global items evaluated satisfaction with HT-I and group dynamics.

In addition to the acceptance questionnaires, students were asked to grade the training on a scale from 1 ("SWIS did not help at all") to 5 ("SWIS helped very much") after completing all training sessions.

\section{Trainer Acceptance Questionnaire}

The TAQ contained seven scales that measured the satisfaction with the sleep training's contents and questions regarding group dynamics (Table 5). The four scales content, hypnotherapy, trainer aptitude and group dynamics were used to measure the trainers' acceptance.

A short section for notes and observations provided opportunity for the documentation of additional information and suggestions regarding the sessions' content.

At the end of the TAQ, the trainers assessed the timeframe, participant comprehension and participant acceptance for each part of the training sessions. The answers were coded in grades from 1 ("completely applies to me") to 5 ("does not apply to me at all”). 
Table 4 Trainer acceptance questionnaire

\begin{tabular}{|c|c|c|}
\hline Scale & No & Items \\
\hline \multirow[t]{4}{*}{ Content (al) } & I & The topic was accepted by the group \\
\hline & 2 & Today's content seemed useful \\
\hline & 3 & $\begin{array}{l}\text { The participants understood the } \\
\text { theoretical knowledge }\end{array}$ \\
\hline & 4 & $\begin{array}{l}\text { The participants understood the practical } \\
\text { knowledge }\end{array}$ \\
\hline \multirow[t]{4}{*}{ Frame conditions ( $\mathrm{fl}$ ) } & 5 & The session's timeframe was adequate \\
\hline & 6 & The session room was adequate \\
\hline & 7 & The time of day was selected appropriately \\
\hline & 10 & The session progressed fluently \\
\hline \multirow[t]{3}{*}{ Instruction (f2) } & 8 & Trainer instructions were comprehensible \\
\hline & 9 & Trainer instructions were helpful \\
\hline & 11 & I felt well prepared \\
\hline Hypnotherapy (a2) & 12 & $\begin{array}{l}\text { I was able to present the trance session } \\
\text { fluently and in an appropriate tone }\end{array}$ \\
\hline \multirow[t]{5}{*}{ Trainer aptitude (a3) } & 13 & I felt comfortable in the group \\
\hline & 14 & The role of a sleep trainer suits me \\
\hline & 15 & I felt overstrained (-) \\
\hline & 17 & I was able to handle difficulties \\
\hline & 19 & Today's session was fun \\
\hline \multirow[t]{4}{*}{ Group dynamics (a4) } & 16 & The group cooperated well \\
\hline & 18 & $\begin{array}{l}\text { Enough opportunities for group discussion } \\
\text { were provided }\end{array}$ \\
\hline & 20 & $\begin{array}{l}\text { I was able to consider each participant's } \\
\text { individual situation }\end{array}$ \\
\hline & 21 & The group size was adequate \\
\hline \multirow[t]{2}{*}{ Co-trainer (f3) } & 22 & $\begin{array}{l}\text { The cooperation with my co-trainer } \\
\text { worked well }\end{array}$ \\
\hline & 23 & I felt supported by my co-trainer \\
\hline
\end{tabular}

Notes: $(a)=$ acceptance scale; $(f)=$ feasibility scale. Item 15 was reversed $(-)$.

\section{Feasibility}

The feasibility of SWIS was indicated by participant dropout and attendance rates during the training. In order to measure the feasibility from the trainer's point of view, four sections from the TAQ were included: three scales (frame conditions,

Table 5 Items of the SPQ (insomnia/irregular sleep schedule)

\begin{tabular}{|c|c|}
\hline No & Item \\
\hline I & I suffer because I have difficulties initiating sleep \\
\hline 2 & I suffer because I have difficulties maintaining sleep \\
\hline 3 & $\begin{array}{l}\text { I suffer because I wake up in the early morning and } \\
\text { I am not able to return to sleep }\end{array}$ \\
\hline 4 & My sleep disturbance causes significant distress or impairment \\
\hline 5 & My sleep problems occur at least three times per week \\
\hline 6 & My sleep problems last longer than I month \\
\hline 7 & I sleep at least three times in 24 hours \\
\hline 8 & Daytime sleepiness reduces my quality of life \\
\hline 9 & I voluntarily (ie, not due to shift work) sleep in irregular patterns \\
\hline 10 & $\begin{array}{l}\text { My sleep problems are not mainly caused by bad sleep } \\
\text { hygiene (ie, bright light) }\end{array}$ \\
\hline II & $\begin{array}{l}\text { My sleep problems are not mainly caused by substance } \\
\text { abuse (ie, alcohol) }\end{array}$ \\
\hline 12 & My sleep problems are not mainly caused by pharmaceuticals \\
\hline
\end{tabular}

\section{Actigraphy}

The SOMNOwatch ${ }^{\mathrm{TM}}$ plus by SOMNOmedics (Randersacker, Germany) is an actigraph wrist watch with three built-in activity sensors. Analysis with the DOMINO Light Software (SOMNOmedics) provides data for time in bed, sleep duration, wake duration, number of night-time awakenings, sleep efficiency and sleep latency. In a study with sleep apnea patients, the SOMNOwatch ${ }^{\mathrm{TM}}$ plus had an overall accuracy of $85.9 \%$ when compared to polysomnography. ${ }^{40}$ The participants wore the actigraphs for 8 days during preand post-measurement.

\section{Epworth Sleepiness Scale}

The ESS ${ }^{41}$ consists of eight items and measures daytime sleepiness across different situations (ie, sitting and reading). 
Despite its relatively short form, both the test-retest reliability $(r=0.82)$ and the internal consistency in clinical $(\alpha=0.88)$ and nonclinical populations $(\alpha=0.73)$ are adequate. ${ }^{42}$

\section{Morningness-Eveningness Questionnaire}

The chronotype was measured with the MEQ. ${ }^{43}$ The MEQ consists of 19 items that add up to a sum score. According to the sum score, participants are classified as definite evening types (14-30), moderate evening types (31-41), neutral type (42-58), moderate morning type (59-69) or definite morning type (70-86). The reliability and validity of the MEQ could be proven, notwithstanding the instruments' multidimensionality. ${ }^{44}$

Fragebogen zur Erfassung spezifischer Persönlichkeitsmerkmale Schlafgestörter (Questionnaire of specific personality traits for patients with sleep disorders)

The FEPS-II ${ }^{45}$ records two personality traits on a five-point Likert scale: focusing (11 items) and rumination (12 items). Gender-specific norms are provided for healthy sleepers $(\mathrm{N}=346)$ and patients with sleep disorders $(\mathrm{N}=322)$. Though the psychometric qualities of the FEPS-II have not been sufficiently examined yet, it is the only instrument that measures these specific personality traits in participants with sleep problems.
Meinungen-zum-Schlaf-Fragebogen (cognitions about sleep) Weingartz and Pillmann developed the MZS in order to investigate the participants' beliefs concerning sleep. ${ }^{46}$ Sixteen convictions about sleep are rated on a ten-point scale ranging from "I absolutely disagree" (0) to "I absolutely agree" (10), with higher scores indicating more dysfunctional beliefs about sleep. A factor analysis by Weingartz and Pillmann revealed the three factors, "convictions about longterm consequences", "biological causes for sleep disorders" and "influence on daytime productivity". According to the authors, the MZS is a reliable $(\alpha=.87)$ and valid instrument. ${ }^{46}$

\section{Intervention: SWIS}

SWIS is a multicomponent group treatment and consists of six training sessions of 100 minutes (Table 6). Behavior therapeutic elements are rules of healthy sleeping, sleeppromoting living arrangements, sleep rituals, progressive muscle relaxation, cognitive restructuring, stress management and problem-solving skills. Trance sessions, self-calming imaginations, self-hypnosis and further imagination techniques comprise the hypnotherapeutic elements. Based on the special requirements and situations of college/university students such as chronotype, sleep environment, self-organization processes for learning and changing starting times at university,

Table 6 Short description for each SWIS session

\begin{tabular}{|c|c|c|}
\hline SWIS & Content & Training at home \\
\hline \multirow[t]{5}{*}{1} & Instructions & Apply two rules of healthy sleeping \\
\hline & Psychoeducation I: healthy sleep, sleep disorders, studying and sleep & Practice HT-I I \\
\hline & Psychoeducation II: model for the development and maintenance of sleep disorders & \\
\hline & Rules for healthy sleeping & \\
\hline & HT-I I: branch in the river (problem solving, promotes change-willingness) & \\
\hline \multirow[t]{5}{*}{2} & Psychoeducation III: vicious-circle model & (Change living arrangements) \\
\hline & Sleep-promoting living arrangements & Practice PMR \\
\hline & Sleep rituals & Practice HT-I 2 \\
\hline & Relaxation: PMR & \\
\hline & HT-I 2: sleep tree (enhance melatonin release) & \\
\hline \multirow[t]{4}{*}{3} & Stress management I: stress multiplier & Practice positive phrases \\
\hline & Cognitive restructuring I: positive and negative phrases & Practice calm picture \\
\hline & Calm picture & Practice HT-I 3 \\
\hline & HT-I 3: rucksack (disengaging oneself from unnecessary ballast) & \\
\hline \multirow[t]{4}{*}{4} & Stress management II: Stress-O-Meter & Apply systematic problem solving to one bigger problem \\
\hline & Systematic problem solving (nightmares) & Practice self-hypnosis \\
\hline & Self-hypnosis & Practice HT-I 4 \\
\hline & HT-I 4: learning in your sleep (improve learning) & \\
\hline \multirow[t]{3}{*}{5} & Psychoeducation IV: situation, perception, behavior and consequences & Practice positive feedback loops \\
\hline & Cognitive restructuring II: positive and negative feedback loops & Practice HT-I 5 \\
\hline & HT-I 5: sleep-wakeland (function of circadian rhythm) & \\
\hline \multirow[t]{4}{*}{6} & Stress management III: useful inner phrases for stress reduction & Practice HT-I \\
\hline & Sleep in exam periods & \\
\hline & Emergency sleep kit & \\
\hline & HT-I 6: red balloon (stress reduction and management) & \\
\hline
\end{tabular}

Abbreviations: SWIS, Studieren wie im Schlaf (studying in your sleep); HT-I, Hypnotherapy for Insomnia; PMR, progressive muscle relaxation. 
first relationship, partying, etc, the treatment was adapted from adolescent treatment (JuSt) and insomnia treatment for adults. Beyond CBT-I, HT-I elements were included to address relaxation and nightmare strategies as imaginary rescripting strategy. As prior results showed, HT-I is helpful to enhance deep sleep and to address insomnia. ${ }^{47,48}$ Participants have to practice the session contents at home.

At the beginning of each session, students were asked to describe their progress and difficulties regarding the implementation of SWIS during everyday life. At the end, they received a CD with the session's HT-I elements to establish practice at home.

\section{Data analysis}

Data were analyzed with the Statistical Package for Social Sciences version 22 (Version 22.0; IBM Corporation, Armonk, NY, USA) and Microsoft Excel 2013 (Microsoft Corporation, Redmond, WA, USA). The inclusion criteria, acceptance questionnaires and grades were analyzed with a descriptive and frequency analysis. The notes and observations of the acceptance questionnaires were listed.

Prior to analysis, each variable was tested for normal distribution (Shapiro-Wilk). The differences between pre- and post-measurement were analyzed with a Student's $t$-test for dependent samples if they were normally distributed and a Wilcoxon signed-rank test if they were not. Effect sizes were Cohen's $d$ for the Student's $t$-test (small $>0.20$, medium $>0.50$, large $>0.80$ ) and Pearson's correlation coefficient $r$ for the Wilcoxon signed-rank test (small $>0.10$, medium $>0.30$, large $>0.50$ ). They were only calculated if the test statistic provided significant $P$-values.

Average weekly sleep duration and regeneration scores were compiled from the diagnostic sleep logs. Missing values were replaced with the average weekly score.

Control for group disparities could not be conducted due to the small sample sizes for pre- and post-measurement comparison. For data analysis, it was assumed that the four treatment groups did not differ significantly.

The significance level was set at $P<0.05$. A tendency was detected with a $P$-value $<0.10$. Due to the small sample size, the missing questionnaire data were not imputed but excluded from the analyses.

\section{Results}

\section{Prevalence rates for sleep problems and diagnoses}

Many participants (74\%) fulfilled criteria for an insomnia disorder or reported various insomnia symptoms in the
SPQ (items 1-3). Eighteen participants (67\%) rated their sleep problems as a strong impairment (item 4). Similarly, 13 participants (48\%) reported that daytime sleepiness reduced their quality of life. Sleep problems often lasted longer than 1 month (74\%) and often occurred more than three times per week $(59 \%)$. Irregular sleep patterns were found in $11 \%$, while fragmented sleep was not reported. Table 7 shows the prevalence rates for each sleep disorder separately. At postmeasurement, fewer students reported sleep disturbances and fulfilled the diagnostic criteria for insomnia and nightmares.

Figure 2 displays information about the comorbidities in the current sample. Most participants displayed sleep disturbances (74\%) or fulfilled the criteria for one sleep disorder (30\%). Insomnia and nightmares were the most common comorbidities (11\%), followed by a combination of all three sleep disorders (7\%). Only 4\% reported insomnia and an irregular sleep-wake type simultaneously. The combination of nightmares and irregular sleep-wake type was not present in this sample.

PSQI sum scores indicated a poor sleep quality mean for the whole group $(\mathrm{M}=10.41, \mathrm{SD}=3.51)$ with a minimum score of six and a maximum score of 18 (Figure 3 ). Therefore, all participants reported a poor sleep quality according to the PSQI. Fifteen participants (55.56\%) even reported a severely impaired sleep quality (PSQI $>10$ ).

\section{Acceptance}

\section{Student Acceptance Questionnaire}

The results for the students' acceptance of the SWIS training as a multicomponent group treatment are presented in Table 8. All SWIS components were well accepted. The scale group dynamics received the highest acceptance ratings, while the participants' acceptance of the HT-I components was only medium.

In accordance with the acceptance questionnaires, students gave an average grade rating of $\mathrm{M}=2.61(\mathrm{SD}=0.69)$ indicating an overall good satisfaction with the SWIS training. Further acceptance was also documented by the fact that only

Table 7 Prevalence rates of sleep disturbances for pre- and postmeasurement

\begin{tabular}{lll}
\hline Sleep disturbances & $\begin{array}{l}\text { Pre-measurement } \\
\text { (\%) }\end{array}$ & $\begin{array}{l}\text { Post-measurement } \\
\text { (\%) }\end{array}$ \\
\hline Difficulties initiating sleep & 59.3 & 37.0 \\
Difficulties & 59.3 & 40.7 \\
maintaining sleep & & \\
Early awakening & 51.9 & 37.0 \\
Subjective impairment & 66.7 & 48.1 \\
Insomnia disorder & 51.9 & 44.4 \\
Nightmares & 18.5 & 7.4 \\
Irregular sleep-wake type & 11.1 & 11.1 \\
\hline
\end{tabular}




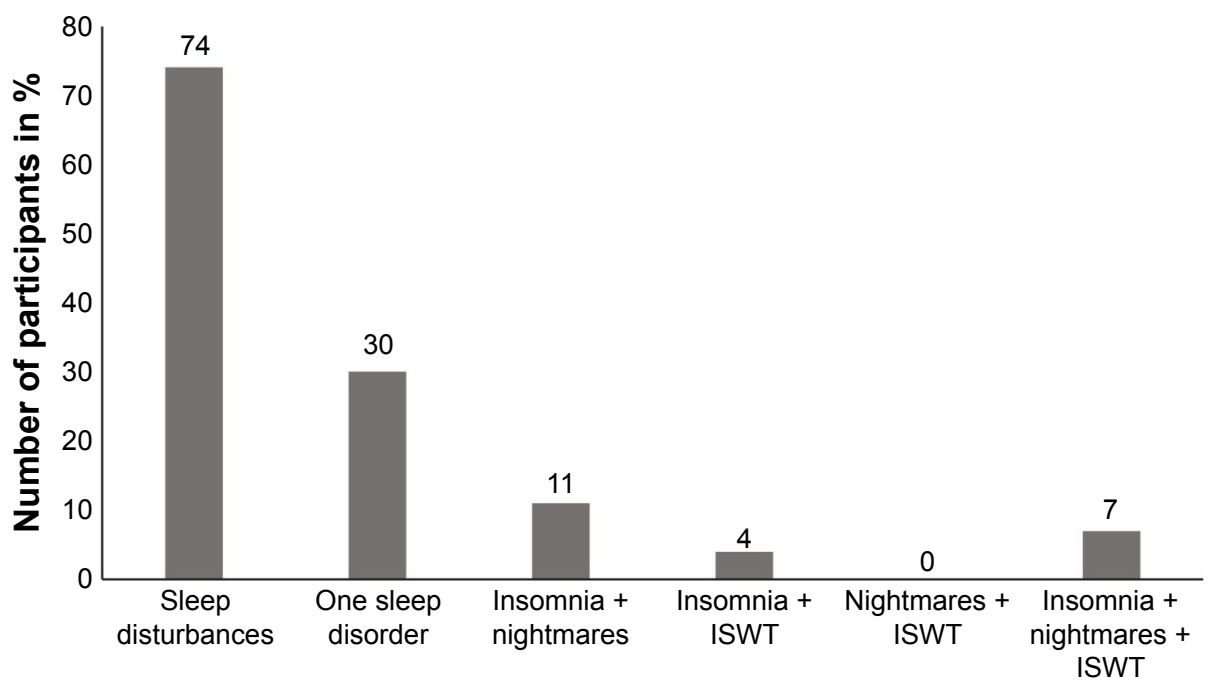

Figure 2 Comorbid sleep disorders. Sleep disturbances indicate difficulties initiating sleep or maintaining sleep, or early awakening. Abbreviation: ISWT, irregular sleep-wake type.

three of the 27 participants discontinued the SWIS program after two sessions. The students' acceptance was also documented by the fact that all participants who completed the program attended at least four of the six sessions.

\section{Trainer Acceptance Questionnaire}

The results for the trainer's acceptance and the feasibility of the SWIS training are demonstrated in Table 9. All scales were evaluated positively. The scale group dynamics received the highest acceptance rating $(\mathrm{M}=1.52, \mathrm{SD}=0.46)$, while the scale co-trainer received the highest feasibility rating $(\mathrm{M}=1.03, \mathrm{SD}=0.13)$.

\section{Feasibility}

The results for the three feasibility scales of the TAQ are presented in Table 9. All scales received favorable evaluation. The scale co-trainer had the best evaluation $(\mathrm{M}=1.03, \mathrm{SD}=0.13)$. Trainers reported a moderate-to-excellent satisfaction with the session parts' timeframe. Mean timeframe ratings ranged

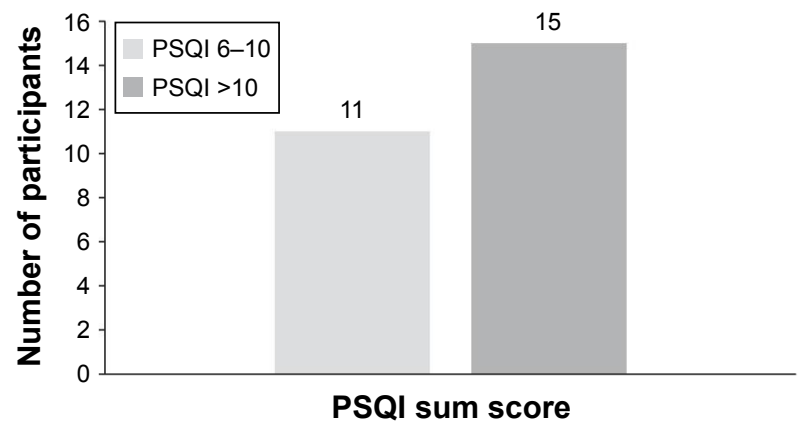

Figure 3 Included participants categorized according to PSQI cut-off scores. Abbreviation: PSQI, Pittsburgh Sleep Quality Index. from excellent for the self-hypnosis in session $4(\mathrm{M}=1.00$, $\mathrm{SD}=0.00)$ to moderate for the rules of healthy sleeping in session $1(\mathrm{M}=2.25, \mathrm{SD}=1.41)$. The trainers evaluated the participants' comprehension of the session parts to be excellent for the feedback elements at the beginning of each session $(\mathrm{M}=1.33, \mathrm{SD}=0.58)$ and moderate for the stress section in session $4(\mathrm{M}=2.50, \mathrm{SD}=0.55)$. The trainers rated the participants' acceptance as excellent for the emergency sleep kit in session $6(\mathrm{M}=1.20, \mathrm{SD}=0.45)$ and moderate for the useful inner phrases in the same session $(\mathrm{M}=3.00, \mathrm{SD}=0.71)$.

\section{Sleep measurements}

The following paragraphs refer to the comparison between pre- and post-measurement.

\section{Pittsburgh Sleep Quality Index}

The PSQI sum scores declined significantly from pre- to postmeasurement (Table 10), indicating a higher sleep quality after the training. Nonparametric results for the seven PSQI scales showed significantly improved scores regarding

Table 8 Students' acceptance of the SWIS training

\begin{tabular}{lll}
\hline & M & SD \\
\hline Content & 2.13 & 0.74 \\
Didactics & 1.74 & 0.53 \\
Transfer & 2.03 & 0.79 \\
HT-I & 2.53 & 1.03 \\
Trainer aptitude & 1.72 & 0.77 \\
Group dynamics & 1.52 & 0.62 \\
\hline
\end{tabular}

Notes: Means and standard deviations are provided for the four scales and two global items. Lower scores represent a higher satisfaction (range I-5).

Abbreviations: SWIS, Studieren wie im Schlaf (studying in your sleep); M, mean; SD, standard deviation; HT-I, Hypnotherapy for Insomnia. 
Table 9 Trainers' acceptance and feasibility of the SWIS training

\begin{tabular}{lll}
\hline & M & SD \\
\hline Acceptance & & \\
Content & 1.71 & 0.46 \\
HT-I & 1.55 & 0.67 \\
Trainer aptitude & 1.66 & 0.51 \\
Group dynamics & 1.52 & 0.46 \\
Feasibility & & \\
Frame conditions & 1.53 & 0.38 \\
Instruction & 1.50 & 0.51 \\
Co-trainer & 1.03 & 0.13 \\
\hline
\end{tabular}

Notes: Means and standard deviations are provided for six scales and one global item. Lower scores represent a higher satisfaction (range I-5).

Abbreviations: SWIS, Studieren wie im Schlaf (studying in your sleep); M, mean; SD, standard deviation; HT-I, Hypnotherapy for Insomnia.

subjective sleep quality, sleep-onset latency and daytime sleepiness. Positive tendencies $(P<0.10)$ were detected for sleep duration and sleep efficiency. The two subscales sleep disturbances and sleep medication did not change significantly according to PSQI. The effect size for the change in the PSQI sum scores was large. The differences in the subscales sleep quality, sleep latency, sleep efficiency and daytime sleepiness reached medium effect sizes, and the scale sleep duration had a small effect.

\section{Sleep logs}

The results for the diagnostic sleep logs are presented in Table 11. Compared to pre-measurement level, the SWIS participants reported a reduced number of night-time awakenings at post-measurement with a medium effect size $(d=0.67)$. The remaining subjective sleep variables from the diagnostic sleep log did not change significantly.

\section{Actigraphy}

Descriptive statistics and differences between pre- and postmeasurement for the actigraphs are displayed in Table 12 . Statistically significant tendencies were discovered for sleep-onset latency, which decreased from 38.4 minutes to
13.8 minutes. This equates to a medium effect size $(r=-0.31)$. Additionally, clinically significant improvements were found for duration of night wakings ( $<30$ minutes at posttest) and sleep efficiency ( $>95 \%$ at posttest).

\section{Epworth Sleepiness Scale}

Average daytime sleepiness decreased from pre- $(M=9.92$, $\mathrm{SD}=5.02)$ to post-measurement $(\mathrm{M}=8.25, \mathrm{SD}=4.83)$, although the difference was not significant $(t(18)=0.86, P=0.40)$.

\section{Morningness-Eveningness Questionnaire}

The average chronotype was neutral during pre- $(\mathrm{M}=45.42$, $\mathrm{SD}=10.34)$ and post-measurement $(\mathrm{M}=47.75, \mathrm{SD}=8.36)$. A nonsignificant development toward morningness was detected $(t(11)=-1.69, P=0.12)$.

Fragebogen zur Erfassung spezifischer Persönlichkeitsmerkmale Schlafgestörter (Questionnaire of specific personality traits for patients with sleep disorders)

The participants' tendency to ruminate decreased significantly $(Z(19)=-2.60, P=0.009, r=-0.42)$ from pre- $(\mathrm{M}=46.53$, $\mathrm{SD}=9.95)$ to post-measurement $(\mathrm{M}=40.37, \mathrm{SD}=11.18)$. In addition, participants reported significantly reduced focusing $(t(18)=4.69, P=0.000, d=1.33)$ after the training $\left(\mathrm{M}_{\mathrm{pre}}=33.00\right.$, $\left.\mathrm{SD}_{\text {pre }}=5.80 ; \mathrm{M}_{\text {post }}=25.63, \mathrm{SD}_{\text {post }}=7.85\right)$.

\section{Meinungen-zum-Schlaf-Fragebogen (cognitions about sleep)}

Descriptive statistics and differences between pre- and postmeasurement for the convictions about sleep are displayed in Table 13. The MZS sum score did not change significantly from pre- to post-measurement. Similar results were found for the two subscales convictions about long-term consequences and biological causes for sleep disorders. However, the participants reported a reduction of dysfunctional beliefs concerning daytime productivity with a small effect size $(d=0.32)$.

Table I0 PSQI: descriptive statistics and comparisons for pre- and post-measurement

\begin{tabular}{|c|c|c|c|c|c|c|c|}
\hline & tl, M (SD) & t2, M (SD) & $\mathbf{N}$ & $t$ & $\mathbf{Z}$ & $P$-value & Effect size, $d / r$ \\
\hline PSQI sum score & $10.7 I^{\mathrm{a}}(3.69)$ & $8.24(3.47)$ & 19 & 3.78 & - & 0.001 & 0.97 \\
\hline Sleep quality & $2.00(0.7 I)$ & $\mathrm{I} .53(0.72)$ & 19 & - & $-2.7 \mathrm{I}$ & 0.007 & -0.44 \\
\hline Sleep latency & $2.11(0.76)$ & $1.65(0.86)$ & 19 & - & -1.99 & 0.046 & -0.46 \\
\hline Sleep duration & I.I8 (1.07) & $0.88(0.86)$ & 19 & - & -1.67 & $0.096^{b}$ & -0.27 \\
\hline Sleep efficiency & I.6I (I.29) & $1.22(1.06)$ & 18 & - & -1.93 & $0.053^{b}$ & -0.32 \\
\hline Sleep disturbances & I.35 (0.49) & $\mathrm{I} .29(0.47)$ & 18 & - & -0.45 & 0.655 & - \\
\hline Sleep medication & $0.47(1.00)$ & $0.29(0.77)$ & 19 & - & -1.63 & 0.102 & - \\
\hline Daytime sleepiness & $\mathrm{I} .88(0.78)$ & $\mathrm{I} .47(0.79)$ & 19 & - & -2.27 & 0.023 & -0.37 \\
\hline
\end{tabular}

Notes: Significant differences $(P<0.05)$ are displayed in bold characters. Higher values indicate worse sleep quality. ${ }^{\mathrm{a}}$ The PSQI sum score differs from the inclusion criteria for the pre-measurement because only participants who completed pre- and post-measurement were included. ${ }^{\mathrm{b}} \mathrm{Tendencies}(P<0.10)$.

Abbreviations: PSQI, Pittsburgh Sleep Quality Index; M, mean; SD, standard deviation. 
Table I I Descriptive statistics of and comparisons between pre- and post-measurement of the diagnostic sleep logs. Regeneration scores are displayed on a six-point Likert scale $(\mathrm{N}=13)$

\begin{tabular}{lllllll}
\hline & tI, M (SD) & t2, M (SD) & $\boldsymbol{t}$ & $\boldsymbol{Z}$ & $\boldsymbol{P}$-value & Effect size, d/r \\
\hline Time in bed (hours) & $8.44($ I.26) & $8.45(I .30)$ & -0.08 & - & 0.94 & - \\
Sleep duration (hours) & $6.94(1.45)$ & $6.83(1.18)$ & 0.57 & - & 0.58 & - \\
Duration of night waking (minutes) & $89.96(49.86)$ & $97.43(53.91)$ & -0.59 & - & 0.563 & - \\
Sleep-onset latency (minutes) & $52.71(54.06)$ & $45.15(35.13)$ & - & -1.06 & 0.289 & - \\
Sleep efficiency (\%) & $83.77(9.69)$ & $81.49(9.28)$ & - & -0.035 & 0.972 & - \\
Number of night-time awakenings & $3.45(0.89)$ & $2.60(1.55)$ & 2.19 & - & $\mathbf{0 . 0 4 9}$ & $\mathbf{0 . 6 7}$ \\
Regeneration & $2.57(0.89)$ & $2.71(0.79)$ & - & -1.36 & 0.17 & - \\
\hline
\end{tabular}

Notes: Duration of night waking describes the discrepancy between time in bed and the sleep duration. Significant differences $(P<0.05)$ are displayed in bold characters. Higher regeneration scores indicate a better regeneration. ${ }^{\mathrm{a}}$ Tendencies $(P<0.10)$.

Abbreviations: $M$, mean; SD, standard deviation.

\section{Discussion}

The purpose of the current study was to examine the feasibility of the SWIS sleep training, a specialized treatment for college/university students. It addresses insomnia symptoms, nightmares and irregular sleep-wake cycles. First indications of acceptance, feasibility and effectiveness were investigated with several instruments including sleep logs, actigraphy and questionnaires.

Overall, participants showed a high acceptance of the SWIS sleep training that encompassed nearly all subscales. The CBT-I elements were rated as very good, whereas the HT-I elements received a slightly lower but still acceptable rating. On average, participants graded the training as good to moderate. The trainers displayed a high to very high acceptance of the SWIS content, hypnotherapy, trainer aptitude and group dynamics. Based on these results, the first hypothesis can be confirmed.

The training's feasibility was reflected in the participants' high attendance and low dropout rates. Similarly, the trainers evaluated the frame conditions, instructions and co-trainers positively, likewise indicating SWIS feasibility from the trainers' perspective.

After the SWIS training, sleep improved on nearly all outcome measures. Students reported a significantly better sleep quality, including shorter sleep latency and less daytime sleepiness according to the PSQI. The sleep log data showed a reduced number of night-time awakenings after the treatment. As an objective measure, actigraphy results showed tendencies of reduced sleep-onset latency and clinically significant improvements for the duration of night wakings and sleep efficiency. Daytime sleepiness according to the ESS and the chronotype did not change significantly from pre- to post-measurement. However, participants showed cognitive changes as a reduction of rumination and focusing as well as a tendency to have less dysfunctional beliefs about daytime productivity. All in all, the students' sleep was improved after the SWIS training.

In general, prior to treatment, the students reported an impaired sleep quality and sleep problems, most commonly insomnia symptoms. This is in accordance with current research that indicates reduced sleep quality and more sleep disorders in university students in comparison to the general population. $^{3}$

After SWIS, the sleep-onset latency decreased in both actigraphy results and subjective ratings (PSQI), which is in accordance with Lichstein et al. ${ }^{49}$ Most notable, sleep was improved on a subjective basis in sleep questionnaires, but also on more detailed instruments as sleep logs and objective actigraphy. However, some instruments that measured the same aspects of sleep did not concur. These differences might

Table 12 Actigraphy: descriptive statistics and comparisons between pre- and post-measurement in hours. Sleep efficiencies are displayed in percentages $(\mathrm{N}=15)$

\begin{tabular}{|c|c|c|c|c|c|c|}
\hline & tI, M (SD) & t2, M (SD) & $t$ & $\mathbf{Z}$ & $P$-value & Effect size, $d / r$ \\
\hline Time in bed (hours) & $8.38(1.28)$ & $8.29(1.18)$ & - & -3.41 & 0.773 & - \\
\hline Sleep duration (hours) & $7.60(1.18)$ & $7.91(1.09)$ & -1.28 & - & 0.221 & - \\
\hline Duration of night waking (minutes) & $46.80(43.20)$ & $22.20(17.40)$ & - & -1.17 & 0.244 & - \\
\hline Sleep-onset latency (minutes) & $38.40(47.40)$ & $13.80(16.20)$ & - & -1.68 & $0.094^{a}$ & -0.31 \\
\hline Sleep efficiency (\%) & $91.56(7.34)$ & $95.52(3.11)$ & - & -1.14 & 0.256 & - \\
\hline Number of night-time awakenings & $29.13(24.56)$ & $18.27(12.63)$ & - & -1.29 & 0.195 & - \\
\hline
\end{tabular}

Notes: Duration of night waking describes the discrepancy between time in bed and the sleep duration. Clinically significant results are displayed in bold. ${ }^{\mathrm{a} T e n d e n c i e s}$ $(P<0.10)$.

Abbreviations: $M$, mean; SD, standard deviation. 
Table 13 MZS: descriptive statistics and comparisons between pre- and post-measurement $(\mathrm{N}=19)$

\begin{tabular}{lllllll}
\hline & tI, M (SD) & t2, M (SD) & t & Z & P-value & Effect size, d/r \\
\hline MZS sum & $84.68(14.95)$ & $78.21(21.39)$ & 1.68 & - & 0.110 & - \\
Dysfunctional beliefs about long-term consequences & $48.68(9.18)$ & $44.63(13.83)$ & 1.70 & - & 0.106 & - \\
Dysfunctional beliefs about biological causes & $11.16(7.21)$ & $11.16(5.88)$ & - & -0.10 & 0.917 & - \\
Dysfunctional beliefs about daytime productivity & $24.84(5.98)$ & $22.42(7.57)$ & 1.89 & - & $0.074^{\mathrm{a}}$ & 0.32 \\
\hline
\end{tabular}

Note: ${ }^{\text {TTendencies }}(P<0.10)$.

Abbreviations: MZS, Meinungen-zum-Schlaf-Fragebogen (cognitions about sleep); M, mean; SD, standard deviation.

be explained by the fact that students with sleep disorders, especially insomnia, often have a distorted perception of sleep. This misperception leads to an overestimation of their sleep-onset latency and an underestimation of their sleep duration. ${ }^{50}$ Disparities between the ESS and the daytime sleepiness from the PSQI can be explained by the different accuracy levels. While the PSQI uses only one item to measure daytime sleepiness, the ESS is a more comprehensive scale with seven items. In any case, daytime sleepiness is more likely to change in patients who suffer from other sleep disorders such as obstructive sleep apnea or narcolepsy than in patients with insomnia. ${ }^{51}$ The lack of significant changes concerning the students' chronotypes may be attributable to the fact that the chronotype is a relatively stable construct in young adults and that changes might need more time. ${ }^{52}$

\section{Cognitive Behavioral Therapy for Insomnia}

The content of the SWIS sleep training including CBT-I and HT-I converges with other programs regarding stimulus control, sleep hygiene, relaxation training and cognitive therapy. Based on theoretical constructions, the reflexion of our training is in line with the results of Taylor et al: ${ }^{53}$ both trainings were accepted by the participants and improved sleep quality as well as sleep latency. Despite these similarities, considerable differences between the CBT-I implemented by Taylor and SWIS emerge. While SWIS does not contain bedtime restriction, it offers a great variety of study-related strategies that are not present in other sleep treatments. These strategies include a focus on stress management, sleep in exam periods, problem solving and the HT-I elements.

In contrast to Taylor et al, the SWIS treatment led to improvements in objective sleep measures (actigraphy), like a reduced duration of night wakings, reduced sleep onset latency and higher sleep efficiency. Furthermore, the participants of the SWIS training reported an improved daytime sleepiness according to PSQI. On the one hand, the differences in daytime sleepiness may be caused by methodical disparities, as Taylor et al used a specific questionnaire to assess daytime sleepiness (ESS), while the current study used the ESS as well as the PSQI subscale. On the other hand, a study that investigated side effects of psychotherapy found that sleep restriction results in objectively reduced total sleep time, increased daytime somnolence and impaired vigilance. ${ }^{54}$ Therefore, the additional positive effects of SWIS on objective measures and daytime sleepiness may be attributable to the elements that are missing (ie, stress management) as well as the elements that are included in Taylor's CBT-I (ie, bedtime restriction).

Most important, the CBT-I concept of Taylor was designed to treat only insomnia, while the SWIS sleep training treats insomnia disorder, nightmares and irregular sleep-wake type according to DSM-5, ${ }^{34}$ enabling a wider range of implementation and effectiveness.

\section{Strengths and limitations}

The most important advantages of the SWIS sleep training are the great applicability to different sleep disorders and the focus on university students' lives. Methodological strengths include the additional application of objective measures of sleep and the anonymity of the acceptance assessments.

As this study was a pilot study, the most obvious limitation is the small sample size of only 27 participants. Two aspects relativize this limitation. Firstly, the current study is a feasibility study with the aim of testing the program's acceptance and seeking first indications of effectiveness. Furthermore, despite the small sample size, significant results for all hypotheses were obtained. Another factor is the quasi-experimental design. Due to the missing control group, the positive effects may be attributable to other factors than SWIS. Finally, the lack of a follow-up measurement impeded the investigation of long-term effects.

\section{Conclusion and future prospects}

The current feasibility study showed a high acceptance and effectiveness of the SWIS sleep training for university students. The SWIS sleep training scored well on participant and trainer acceptance as well as feasibility. Furthermore, the students' sleep quality improved significantly, making it a feasible program for university context. 
These results strongly support further investigation of the SWIS sleep training, especially regarding important aspects of college students' life such as study-related impairments and cognitive performance. These could encompass the ability to concentrate as well as different aspects of memory, learning and other neuropsychological faculties. Future evaluations should include the methodological changes indicated by the limitations section, that is, a control group design, follow-up assessments and a larger sample size to expand the generalization of results. Lastly, the improvements in the sleep variables may lead to reduced mental health issues (ie, depression, anxiety, somatization, etc) and a better life quality, which should be assessed in future studies.

\section{Abbreviations}

CBT-I, Cognitive Behavioral Therapy for Insomnia; DSM-5, Diagnostic and Statistical Manual of Mental Disorders (fifth edition); ESS, Epworth Sleepiness Scale; FEPS-II, Fragebogen zur Erfassung spezifischer Persönlichkeitsmerkmale Schlafgestörter (Questionnaire of specific personality traits for patients with sleep disorders); GPA, grade point average; HT-I, Hypnotherapy for Insomnia; JuSt, Jugendlichen Schlaftraining (sleep training for adolescents); KiSS, Kinder Schlaftherapie - Studie (childrens' sleep therapy study); M, mean; MEQ, Morningness-Eveningness Questionnaire; MZS, Meinungen-zum-Schlaf-Fragebogen (cognitions about sleep); PSQI, Pittsburgh Sleep Quality Index; SAQ, Student Acceptance Questionnaire; SD, standard deviation; SPQ, Sleep Problems Questionnaire; SWIS, Studieren wie im Schlaf (studying in your sleep); TAQ, Trainer Acceptance Questionnaire.

\section{Acknowledgments}

The authors would like to thank Neele Busse for supporting the diagnostic process and being a co-trainer. Furthermore, they would like to thank Nina Schäfer for helping with the analysis of the actigraphy data. They also acknowledge the support for Article Processing Charge by the Deutsche Forschungsgemeinschaft and the Open Access Publication Fund of Bielefeld University.

\section{Author contributions}

All authors contributed toward data analysis, drafting and critically revising the paper and agree to be accountable for all aspects of the work.

\section{Disclosure}

The authors report no conflicts of interest in this work.

\section{References}

1. Taylor DJ, Bramoweth AD, Grieser EA, Tatum JI, Roane BM. Epidemiology of insomnia in college students: relationship with mental health, quality of life, and substance use difficulties. Behav Ther. 2013; 44(3):339-348.

2. Buboltz WC Jr, Brown F, Soper B. Sleep habits and patterns of college students: a preliminary study. J Am Coll Health. 2001;50(3): 131-135.

3. Lund HG, Reider BD, Whiting AB, Prichard JR. Sleep patterns and predictors of disturbed sleep in a large population of college students. J Adolesc Health. 2010;46(2):124-132.

4. Gaultney JF. The prevalence of sleep disorders in college students: impact on academic performance. J Am Coll Health. 2010;59(2):91-97.

5. Schlarb AA, Kulessa D, Gulewitsch MD. Sleep characteristics, sleep problems, and associations of self-efficacy among German university students. Nat Sci Sleep. 2012;4:1-7.

6. Schlarb AA, Bihlmaier I, Hautzinger M, Gulewitsch MD, Schwerdtle B. Nightmares and associations with sleep quality and self-efficacy among university students. J Sleep Disord Manag. 2015;1(2):1-5.

7. Alapin I, Fichten CS, Libman E, Creti L, Bailes S, Wright J. How is good and poor sleep in older adults and college students related to daytime sleepiness, fatigue, and ability to concentrate? J Psychosom Res. 2000;49:381-390.

8. Trockel MT, Barnes MD, Egget DL. Health-related variables and academic performance among first-year college students: implications for sleep and other behaviors. J Am Coll Health . 2000;49(3):125-131.

9. Curcio G, Ferrara M, De Gennaro L. Sleep loss, learning capacity and academic performance. Sleep Med Rev. 2006;10(5):323-337.

10. Abdel-Khalek AM. The relation between insomnia and chronic fatigue among a non-clinical sample using questionnaires. Sleep Hypn. 2009; 11(1):9-17.

11. Sing CY, Wong WS. Prevalence of insomnia and its psychosocial correlates among college students in Hong Kong. J Am Coll Health. 2010; 59(3):174-182.

12. Taylor DJ, Gardner CE, Bramoweth AD, et al. Insomnia and mental health in college students. Behav Sleep Med. 2011;9(2):107-116.

13. Harvey AG. Sleep hygiene and sleep-onset insomnia. J Nerv Ment Dis. 2000;188(1):53-55.

14. Guastella AJ, Moulds ML. The impact of rumination on sleep quality following a stressful life event. Pers Individ Dif. 2007;42:1151-1162.

15. Pillai V, Steenburg LA, Ciesla JA, Roth T, Drake CL. A seven day actigraphy-based study of rumination and sleep disturbance among young adults with depressive symptoms. J Psychosom Res. 2014; 77(1):70-75.

16. Carney CE, Harris AL, Moss TG, Edinger JD. Distinguishing rumination from worry in clinical insomnia. Behav Res Ther. 2010; 48(6):540-546.

17. Spiegelhalder K, Regen W, Feige B, et al. Sleep-related arousal versus general cognitive arousal in primary insomnia. J Clin Sleep Med. 2012; 8(4):431-437.

18. Takano K, Iijima Y, Tanno Y. Repetitive thought and self-reported sleep disturbance. Behav Ther. 2012;43(4):779-789.

19. Vail-Smith K, Felts WM, Becker C. Relationship between sleep quality and health risk behaviors in undergraduate college students. Coll Stud J. 2009;43(3):924-930.

20. Zschoche M, Schlarb AA. Is there an association between insomnia symptoms, aggressive behavior, and suicidality in adolescents? Adolesc Health Med Ther. 2015;6:29-36.

21. Nadroff MR, Nazem S, Fiske A. Insomnia symptoms, nightmares, and suicidal ideation in a college student sample. Sleep. 2011;34(1):93-98.

22. Lohsoonthorn V, Khidir H, Casillas G, et al. Sleep quality and sleep patterns in relation to consumption of energy drinks, caffeinated beverages, and other stimulants among Thai college students. Sleep Breath. 2013;17(3):1017-1028.

23. Lohmann K, Gusy B, Drewes J. Medikamentenkonsum bei Studierenden. Präv Gesundheitsf. 2010;5(3):276-281. 
24. Lehnkering H, Siegmund R. Influence of chronotype, season, and sex of subject on sleep behavior of young adults. Chronobiol Int. 2007;24(5):875-888

25. Medeiros ALD, Mendes DB, Lima PF, Araujo JF. The relationships between sleep-wake cycle and academic performance in medical students. Biol Rhythm Res. 2001;32(2):263-270.

26. Digdon NL, Howell AJ. College students who have an eveningness preference report lower self-control and greater procrastination. Chronobiol Int. 2008;25(6):1029-1046.

27. Roeser K, Brückner D, Schwerdtle B, Schlarb AA, Kübler A. Healthrelated quality of life in adolescent chronotypes - a model for the effects of sleep problems, sleep-related cognitions, and self-efficacy. Chronobiol Int. 2012;29(10):1358-1365.

28. Roeser K, Meule A, Schwerdtle B, Kübler A, Schlarb AA. Subjective sleep quality exclusively mediates the relationship between morningness-eveningness preference and self-perceived stress response. Chronobiol Int. 2012;29(7):955-960.

29. Roeser K, Schlarb AA, Kübler A. The Chronotype-Academic Performance Model (CAM): daytime sleepiness and learning motivation link chronotype and school performance in adolescents. Pers Individ Dif. 2013;54(7):836-840

30. Friedrich A, Schlarb AA. Let's talk about sleep: a systematic review of psychological interventions to improve sleep in university students. J Sleep Res. Epub 2017 Jun 15.

31. Schlarb AA, Brandhorst I, Hautzinger M. Mini-KiSS - ein multimodales Gruppentherapieprogramm für Eltern von Kleinkindern mit Schlafstörungen. [Mini-KiSS - a multimodal group therapy intervention for parents of young children with sleep disorders: a pilot study] Z Kinder Jugendpsychiatr Psychother. 2011;39(3):197-206. German [with English abstract].

32. Schlarb AA. KiSS - Therapeutenmanual: Das Training für Kinder von 5 bis 10 Jahren mit Schlafstörungen [KiSS - Therapists Manual: The Training for Children from 5 to 10 Years with Sleep Disorders]. Kohlhammer Verlag. APA: Schlarb, A. (2014); 2010. German.

33. Schlarb AA, Liddle CC, Hautzinger M. JuSt - a multimodal program for treatment of insomnia in adolescents: a pilot study. Nat Sci Sleep. 2011;3:13-20.

34. American Psychiatric Association. Diagnostic and Statistical Manual of Mental Disorders. 5th ed. Arlington: American Psychiatric Publishing; 2013.

35. Buysse DJ, Reynolds CF 3rd, Monk TH, Berman SR, Kupfer DJ. The Pittsburgh Sleep Quality Index: a new instrument for psychiatric practice and research. Psychiatry Res. 1989;28(2):193-213.

36. Wittchen HU, Krause P, Höfler M, et al. NISAS-2000 - die "Nationwide Insomnia Screening and Awareness Study" [NISAS-2000 - the "Nationwide Insomnia Screening and Awareness Study"]. Nervenheilkunde. 2001;20:4-16. German [with English abstract].

37. Zeitlhofer J, Schmeiser-Rieder A, Tribl G, et al. Sleep and quality of life in the Austrian population. Acta Neurol Scand. 2000;102(4):249-257.

38. Curcio G, Tempesta D, Scarlata S, et al. Validity of the Italian version of the Pittsburgh Sleep Quality Index (PSQI). Neurol Sci. 2013;34(4):511-519.
39. Carpenter JS, Andrykowski MA. Psychometric evaluation of the Pittsburgh Sleep Quality Index. J Psychosom Res. 1998;45(1):5-13.

40. Dick R, Penzel T, Fietze I, Partinen M, Hein H, Schulz J. AASM standards of practice compliant validation of actigraphic sleep analysis from SOMNOwatch ${ }^{\mathrm{TM}}$ versus polysomnographic sleep diagnostics shows high conformity also among subjects with sleep disordered breathing. Physiol Meas. 2010;31(12):1623-1633.

41. Johns MW. A new method for measuring daytime sleepiness: the Epworth Sleepiness Scale. Sleep. 1991;14(6):540-545.

42. Johns MW. Reliability and factor analysis of the Epworth Sleepiness Scale. Sleep. 1992;15(4):376-381.

43. Horne JA, Ostberg O. A self-assessment questionnaire to determine morningness-eveningness in human circadian rhythms. Int J Chronobiol. 1976;4(2):97-110.

44. Neubauer AC. Psychometric comparison of two circadian rhythm questionnaires and their relationship with personality. Pers Individ Dif. 1992;13:125-131.

45. Hoffmann RM, Schnieder G, Heyden T. Fragebogen zur Erfassung spezifischer Persönlichkeitsmerkmale Schlafgestörter (FEPS-II) [Questionnaire for recording general and specific personality characteristics of sleep disorders]. Handanweisung. Göttingen: Hogrefe; 1996. German.

46. Weingartz S, Pillmann F. Meinungen-zum-Schlaf-Fragebogen. Somnologie. 2009;13(1):29-36.

47. Cordi MJ, Schlarb AA, Rasch B. Deepening sleep by hypnotic suggestion. Sleep. 2014;37(6):1143-1152.

48. Lam TH, Chung KF, Yeung WF, Yu BYM, Yung KP, Ng THY. Hypnotherapy for insomnia: a systematic review and meta-analysis of randomized controlled trials. Complement Ther Med. 2015;23(5):719-732.

49. Lichstein KL, Stone KC, Donaldson J, et al. Actigraphy validation with insomnia. Sleep. 2006;29(2):232-239.

50. Harvey AG, Tang NK. (Mis)perception of sleep in insomnia: a puzzle and a resolution. Psychol Bull. 2012;138(1):77-101.

51. Schneider C, Fulda S, Schulz H. Daytime variation in performance and tiredness/sleepiness ratings in patients with insomnia, narcolepsy, sleep apnea and normal controls. J Sleep Res. 2004;13(4):373-383.

52. Barclay NL, Rowe R, O’Leary R, Bream D, Gregory AM. Longitudinal stability of genetic and environmental influences on the association between diurnal preference and sleep quality in young adult twins and siblings. J Biol Rhythms. 2016;31(4):375-386.

53. Taylor DJ, Zimmermann MR, Gardner CE, et al. A pilot randomized controlled trial of the effects of cognitive-behavioral therapy for insomnia on sleep and daytime functioning in college students. Behav Ther. 2014;45(3):376-389.

54. Kyle SD, Miller CB, Rogers Z, Siriwardena AN, Macmahon KM, Espie CA. Sleep restriction therapy for insomnia is associated with reduced objective total sleep time, increased daytime somnolence, and objectively impaired vigilance: implications for the clinical management of insomnia disorder. Sleep. 2014;37(2):229-237.
Neuropsychiatric Disease and Treatment

\section{Publish your work in this journal}

Neuropsychiatric Disease and Treatment is an international, peerreviewed journal of clinical therapeutics and pharmacology focusing on concise rapid reporting of clinical or pre-clinical studies on a range of neuropsychiatric and neurological disorders. This journa is indexed on PubMed Central, the 'PsycINFO' database and CAS,

\section{Dovepress}

and is the official journal of The International Neuropsychiatric Association (INA). The manuscript management system is completely online and includes a very quick and fair peer-review system, which is all easy to use. Visit http://www.dovepress.com/testimonials.php to read real quotes from published authors. 